\title{
EVOLUCIÓN GRAMATICAL DE LAS CONSTRUCCIONES PROGRESIVAS DEL INGLÉS: UNA DISCUSIÓN DIACRÓNICA*
}

\author{
Carlos van Arkel-Simón \\ Universidad de La Laguna
}

\begin{abstract}
RESUMEN: En este artículo se presenta una discusión sobre la evolución gramatical de las construcciones progresivas del inglés. A partir de una serie de prototipos progresivos extraídos del conjunto de corpus York-Toronto-Helsinki-Penn, se ilustra cómo la configuración léxicogramatical de la construcción progresiva que caracteriza el inglés actual se encuentra ya en el sistema gramatical de la lengua desde el inglés antiguo. Asimismo, se explica cómo el desarrollo sintáctico del inglés posibilita el establecimiento de la obligatoriedad gramatical de esta construcción perifrástica verbal. Por último, se explica cómo los patrones gramaticales y los rasgos semántico-pragmáticos que caracterizan a las construcciones progresivas inglesas a lo largo de su desarrollo aparentemente se manifiestan a través de un gradiente morfosintáctico-funcional y de cambio gramatical.
\end{abstract}

PALABRAS CLAVE: lingüística histórica inglesa, análisis morfosintáctico, gramaticalización, cambio construccional, construccionalización

Grammatical evolution of the English progressive construction: a diachronic discussion

\begin{abstract}
In this paper we bring forward a comprehensive discussion on the grammatical development of the English progressive construction. From a set of progressive prototypes drawn from the family of corpora York-Toronto-Helsinki-Penn, we exemplify how the lexical and grammatical configuration of the construction which is characteristic of Present-Day English appears since Old English. Furthermore, we explain how the syntactic evolution of English enables the establishment of the obligatory grammatical nature of the verbal periphrastic construction. Finally, we explain how the grammatical patterns and the semantic and pragmatic features that describe the English progressive construction in the course of its evolution are manifested apparently through a functional-morphosyntactic and grammatical change gradient.
\end{abstract}

KEYWORDS: English historical linguistics, morphosyntactic analysis, grammaticalization, constructional change, constructionalization

${ }^{*}$ El contenido de este artículo ha sido elaborado a partir del manuscrito de mi tesis doctoral $E l$ cambio gramatical en las construcciones progresivas del inglés (2018). 


\section{INTRODUCCIÓN}

Las construcciones progresivas del inglés constituyen una categoría gramatical cuya interpretación hace necesario un análisis de todos los niveles de la lengua. Diversos factores semántico-pragmáticos y morfosintácticos intervienen de forma determinante en la génesis y evolución de estas estructuras. Así, la evolución de estas formas perifrásticas del inglés presenta un desarrollo único entre las lenguas germánicas ya que, en dichas lenguas, las construcciones similares desaparecen y, en cualquier caso, no presentan en la actualidad un paradigma progresivo tan amplio como el del inglés ( $c f$. Smith, 2007; Killie, 2014; Petré, 2015, 2017; van Arkel-Simón, 2019 y 2020). Lo cierto es que el inglés desarrolla un sistema aspectual único entre las lenguas germánicas para codificar la noción de progresividad, el cual viene dado por un marcado proceso de gramaticalización y, muy probablemente, de cambio construccional y construccionalización en determinadas estructuras del paradigma progresivo. $^{1}$

Como se verá más adelante al ilustrar los patrones progresivos registrados en inglés antiguo (en adelante, IA), inglés medio (en adelante, IM), inglés moderno temprano (en adelante, IMT) e inglés moderno tardío (en adelante, IModT), se constata la presencia de esta construcción aspectual del inglés desde los primeros períodos, pero ¿̇podemos asegurar una continuidad de la construcción desde el IA al IM? O bien: ¿se trata de una construcción, la progresiva, doble o fragmentada? En otras palabras: ¿hay una continuidad en la construcción desde su aparición en IA o, por el contrario, se trata de una evolución vicaria?

Según Núñez-Pertejo (2004, 53-63), la naturaleza de la construcción progresiva verbal en IA consta de la combinación de los auxiliares beon/wesan ('to be') y weorðan ('to become') -los dos primeros con mayor frecuencia de aparición-y la forma verbal terminada en -ende ('-ing'). Otros auxiliares, como cuman ('to come'), sittan ('to sit'), faran ('to go') y geseon ('to see'), también pueden aparecer en combinación con el participio presente.

Asimismo, Núñez-Pertejo (2004, 62-63) mantiene que las formas progresivas en IA, si bien presentan un paradigma restringido, no son infrecuentes. El estudio de Scheffer (1975), basado en análisis de corpus, presenta unas conclusiones similares, aunque el carácter limitado de su compilación de textos no permite obtener resultados concluyentes. NúñezPertejo (2004, 68-73) también subraya la importancia de la relación que existe entre las formas típicamente verbales terminadas en -ende, las formas típicamente nominales en -end, y las formas adjetivales, también en -ende, que se atestiguan en IM. Considérese, a modo de ilustración, los ejemplos siguientes:

\footnotetext{
${ }^{1}$ La gramaticalización es el proceso de cambio lingüístico mediante el cual formas que inicialmente presentan carácter léxico adquieren carácter gramatical y estas últimas, a su vez, adquieren mayor carácter gramatical. En los modelos construccionales, el cambio gramatical se describe mediante el análisis de las variaciones que experimentan los pares simbólicos de forma y significado construccional, lo que puede dar lugar a una reorganización parcial del sistema construccional de la lengua, o bien a la aparición de nuevas construcciones lingüísticas para codificar una categoría gramatical concreta.
} 
(1) a. He wæs ehtend cristenramonna

[He] [was] [(a) persecutor (of)] [Chistian] [men]

b. He wæs ehtende cristenra monna

[He] [was] [persecuting] [Christian] [men]

(Denison, 1993, 372)

(2) Pæt flowende wæter

[the] [runnig] [water]

(Núñez-Pertejo, 2004, 69)

Fisher (1992, 250-256) plantea que la integración de las formas típicamente nominales y los participios en -ing constituye uno de los factores determinantes en el desarrollo de las construcciones progresivas durante el período del inglés medio tardío. Por tanto, puede aventurarse que los patrones preposicionales y la gran variedad de construcciones con el sufijo -ing darán lugar a las estructuras progresivas actuales. Más concretamente, el paradigma flexivo del participio presente y del gerundio tendían a confundirse a finales del IM. En este mismo sentido, los cambios sintácticos que tienen lugar en este período hacen que las formas de gerundio, en -ung, muestren características propias de las formas verbales, como adoptar sintagmas nominales como objeto directo y presentar modificación adverbial. En estos contextos, se observa finalmente que las estructuras propias del IA, como he was huntende y he was on huntunge, experimentan coalescencia para dar lugar a las formas he was huntyng(e) y he was on/ an/ in/ a huntyng(e).

Las construcciones progresivas del IM también se asemejan mucho a las actuales en cuanto a su estructura, es decir, encontramos la forma del verbo to be junto al participio presente con el sufijo -ing. Con todo, en IM resulta de especial importancia la variación morfológica de las formas en -ing: -ende, -ande, -inde, -inge, -ing.

En cualquier caso, resulta asimismo fundamental relacionar estos patrones con los procesos de cambio gramatical que influyen en el desarrollo de la construcción en cada período. Así, se ha evidenciado que los fenómenos de gramaticalización, cambio construccional y construccionalización son los que permiten explicar los patrones evolutivos de estas construcciones ( $c f$. Núñez-Pertejo, 2004; Smitterberg, 2005; Kranich, 2010).

En primer término, Kranich $(2010,77)$ mantiene que las construcciones progresivas del inglés moderno básicamente funcionan como un marcador aspectual que presenta un alto grado de gramaticalización, lo que contrasta con el resto de las lenguas germánicas actuales, exceptuando el islandés que también presenta esta función marcadamente aspectual.

Asimismo, es bien probable que determinados procesos de cambio construccional y construccionalización hayan tenido lugar a la par de los fenómenos de gramaticalización. En este sentido, determinados procesos característicos de la construccionalización aparentan producirse en el desarrollo de las formas progresivas: lexicalización de la macroconstrucción progresiva para codificar oposiciones semánticas abstractas; es decir, aspecto progresivo frente a no progresivo, así como la redistribución concomitante de las frecuencias relativas de 
aparición de las estructuras y funciones que codifican la categoría aspectual del inglés ( $c f$. Van Arkel-Simón, 2018). ${ }^{2}$

Por tanto, a la luz de estas descripciones, se hace necesaria una explicación de la evolución gramatical de las construcciones progresivas del inglés desde el IA hasta el IModT, la cual englobe los principales hallazgos que se recogen en la literatura especializada relevante.

Así, la discusión que presento en este artículo tiene como objeto explicar los principales patrones morfosintácticos que presentan las construcciones progresivas inglesas a lo largo de los diferentes períodos, más concretamente, desde el IA hasta el IModT, en relación con los procesos de cambio gramatical fundamentales en que devienen estas formas. Para ilustrar esta discusión diacrónica, el cambio lingüístico que se presenta se extrae de los patrones registrados en el conjunto de corpus diacrónicos equiparables York-Toronto-Helsinki-Penn (cf. Kroch y Taylor, 2000; Taylor et al., 2003; Kroch et al., 2004; Kroch, Santorini y Diertani, 2010).

\section{Metodología}

Para ilustrar la discusión que se presenta en este artículo, se parte de los resultados obtenidos del análisis estadístico del conjunto de corpus York-Toronto-Helsinki-Penn, así como del análisis cualitativo de 11.480 ocurrencias progresivas extraídas de dicho conjunto ( $c f$. Van Arkel-Simón, 2018, 2019, 2020).

Por tanto, los prototipos que se presentan se han seleccionado por su idoneidad para ejemplificar los hallazgos obtenidos sobre las características de la construcción progresiva, así como por su capacidad para explicar los procesos de cambio que experimenta la construcción, tras el análisis del conjunto de corpus York-Toronto-Helsinki-Penn.

En primer lugar, los prototipos seleccionados para ilustrar las tendencias observadas en cada período se encuentran contenidos en los géneros textuales que se corresponden con las secciones del conjunto de corpus en las que la confluencia de las variables computables que resultan significativas en los procesos de gramaticalización muestran sus aumentos más acusados.

Así, en el grupo de corpus York-Toronto-Helsinki-Penn, para cada período de la lengua, los géneros textuales que a continuación se indican constituyen el punto de intersección gráfica o convergencia entre los aumentos más acusados de los Coeficientes-M y $-\mathrm{V}$ de las formas progresivas, las variantes sintácticas de estas estructuras que resultan significativas en

\footnotetext{
${ }^{2}$ En la construccionalización, la paradigmatización es fundamental, pues el surgimiento de un nuevo paradigma gramatical se refleja en la aparición y consolidación de una nueva macroconstrucción, la cual configura un nuevo par simbólico de forma y significado. La sucesión de cambios construccionales puede resultar en la construccionalización de la estructura si se genera un nuevo par gramatical de estructura y función a partir de una serie de microconstrucciones intermedias. Estas microconstrucciones presentarán gradualmente mayor carácter gramatical, ya que debe perderse paulatinamente contenido léxico-semántico y fijarse la estructura morfosintáctica asociada al significado final de la macroconstrucción.
} 
diversos modelos predictivos, la ratio clase-tipo léxica progresiva estimada y los cambios más pronunciados en las dimensiones funcionales significativas del modelo multidimensional (MD) en relación con los procesos de gramaticalización ( $c f$. Van Arkel-Simón, 2018):3

1) Homilías para el período del IA.

2) Tratados para el período del IM.

3) Biografía para el período del IMT.

4) Biografía para el período del IModT.

En segundo lugar, para ilustrar los patrones estructurales y funcionales de las construcciones progresivas registrados en las 11.480 ocurrencias analizadas, se emplean una serie de prototipos correspondientes a la clasificación estructural de cada ocurrencia de la construcción según su pertenencia a uno de los tipos básicos de estructuras observadas en el período del inglés contemporáneo (en adelante, IC): verbal, adjetival o nominal. Al mismo tiempo, se procede a identificar en dichos prototipos la presencia o ausencia de las funciones semántico-pragmáticas y rasgos morfosintácticos principales observados en IC. Estos patrones incluyen el conjunto de funciones y estructuras observadas en todos los períodos ( $c f$. Van Arkel-Simón, 2019).

Por último, para explicar los patrones de cambio gramatical en las construcciones progresivas del inglés, se seleccionan una serie de prototipos que permiten la ejemplificación de los rasgos principales correspondientes a los procesos de gramaticalización, tanto primaria como secundaria, así como de los rasgos correspondientes a los fenómenos de cambio construccional y construccionalización según se observa en las 11.480 ocurrencias estudiadas (cf. Van-Arkel-Simón, 2020).

\section{LA EVOLUCIÓN DE LAS CONSTRUCCIONES PROGRESIVAS DEL INGLÉS}

\subsection{La naturaleza de la construcción progresiva en inglés antiguo}

El origen exacto de la construcción progresiva en IA no resulta plenamente claro si no se tienen en cuenta los patrones gramaticales que guardan relación con esta estructura en el resto de las lenguas con las que entra en contacto a lo largo de su existencia. Más específicamente,

3 El Coeficiente-M se utiliza para contabilizar la forma progresiva como rasgo lingüístico autónomo (cf. Smitterberg, 2005, 40). El Coeficiente-V computa el número de formas progresivas con el número de sintagmas verbales sin excluir las formas verbales que no pueden ocurrir en la forma progresiva. (cf. Smitterberg, 2005, 44). Por su parte, la ratio clase-tipo léxica resulta de la división del número distintivo de tipos léxicos progresivos estimados en cada uno de los corpus entre el número total de ocurrencias progresivas extraídas de cada corpus. Por otra parte, para analizar cuantitativamente los cambios en la distribución funcional de las estructuras progresivas puede emplearse el método estadístico MD ( $c f$. Biber, 1988; Biber y Finegan, 1997; Geisler, 2002). Este método básicamente consiste en la construcción de una serie de dimensiones funcionales a partir de características morfosintácticas presentes en los corpus en función del género textual. 
sería deseable analizar la relación existente entre las estructuras progresivas del IA y construcciones similares del galés antiguo, antiguo escocés, antiguo nórdico, francés antiguo y latín vulgar.

La existencia de la construcción progresiva en islandés y danés contemporáneos podría ser indicativa del origen germánico de la misma, tal y como apuntan Visser (1963-1973, § 1852-1855), Nickel (1967, 205-272), Scheffer (1975, 131-206) y Mitchell (1985, § 695-696). La presencia de construcciones seudoprogresivas en el neerlandés y el renano actuales parece indicar que esta construcción tampoco es completamente extraña en las lenguas germánicas occidentales.

Asimismo, no puede obviarse la influencia que debió ejercer el latín en la aparición de estas estructuras en IA. En este sentido, parece innegable la influencia románica en los esfuerzos monásticos por alcanzar un cierto grado de estandarización al copiar y manuscribir los textos en IA. La hipótesis del origen románico de la construcción progresiva inglesa es la que mantienen Jespersen (1931, 165), Mossé (1938, 132-200), Strang (1970, 280-311) y Baugh y Cable (2000, 292).

Tampoco puede descartarse que el sustrato céltico existente en las islas británicas haya favorecido la aparición de esta construcción (Hogg y Denison, 2006, 136). Así, el carácter analítico de las lenguas célticas antiguas pudo favorecer la aparición de esta forma perifrástica en IA. De hecho, en los dialectos del inglés actual de Gales, Escocia e Irlanda parece registrarse una mayor incidencia en el uso de las construcciones progresivas que en el resto de Gran Bretaña ( $c f$. Núñez-Pertejo, 2004, 80). En defensa de la hipótesis del origen céltico, puede argüirse también que el sajón occidental tiende a seleccionar un nominal de carácter verbal tras una preposición en sintagmas preposicionales, y no una forma de infinitivo. Así, los dialectos celtas antiguos parece que hacen uso de este mismo patrón nominal de tipo verbal, puesto que carecen de un paradigma completo en las formas verbales no personales ( $c f$. Dal, 1952, 101-107).

En mi opinión, la preponderancia de la influencia románica, así como las necesidades gramaticales y expresivas concretas que presentaba el IA en su consolidación como lengua vernácula podrían ser las principales causas que motivaron la integración de la construcción progresiva en el sistema gramatical del IA. Así, esta construcción constituía una categoría gramatical que permitía cubrir un espectro de funciones semántico-pragmáticas que empezaban a resultar esenciales en el sistema lingüístico del IA. Esto pudo ocurrir con independencia de la proclividad que seguramente mostraba esta lengua germánica, ya para asimilar, ya para generar estas estructuras en un primer momento, así como independientemente de las influencias que sin duda ejercieron algunas de las lenguas coetáneas anteriormente mencionadas. Con todo, estas necesidades funcionales pueden explicarse a la luz de los destacados procesos de cambio lingüístico en curso durante el período del IA, lo que daría cuenta de una teoría mixta sobre el origen de las construcciones progresivas del inglés que, por otra parte, probablemente sea la más certera.

En primer lugar, el uso de esta estructura perifrástica en IA cobra relevancia al producirse la paulatina desemantización del sistema morfológico aspectual del germánico (en adelante, 
Germ.), el cual empleaba la afijación verbal para denotar distinciones aspectuales. Esta desemantización, tal y como describe Mossé (1938, 11-42), surge del paulatino quebranto en el sistema de oposiciones entre las formas verbales con partícula preverbal y las formas que carecen de ella. Este proceso se completa hacia finales del período del IA, cuando este tipo de prefijación desaparece o presenta un carácter meramente residual en el sistema gramatical del inglés. De este modo, las partículas preverbales como $a-$ - æt-, be-, ge-, on-, for-, under-, purh- y wið- pierden paulatinamente la carga semántica correspondiente al aspecto que es propia del Germ. Específicamente, aunque aún se conserva esta relación de oposición en IA, comienza a diluirse la distinción que confieren las partículas preverbales, especialmente ge-, para expresar un resultado o la ausencia de duración en el proceso denotado por el verbo léxico o de contenido semántico. En otras palabras, estos prefijos se empleaban en Germ. para codificar un proceso perfectivo en contraposición con la duración implícita de las formas que carecían de los mismos, las cuales codifican un proceso imperfectivo: beran ('llevar')/geberan ('proporcionar'), pencan ('pensar')/gepencan ('determinar'), slæpan ('dormir')/geslæpan ('yacer'), etc. En virtud de estas relaciones de oposición, especificadas mediante la presencia o ausencia de una partícula preverbal, se observa que las estructuras progresivas son menos frecuentes en las formas verbales prefijadas en IA ( $c f$. Scheffer, 1975, 178).

En segundo lugar, el desarrollo de otro tipo de estructuras sintagmáticas en IA, como las correspondientes a la conjugación de los tiempos perfectos, pudo asimismo favorecer la integración de la construcción progresiva en este período del inglés ( $c f$. Traugott, 1992, 188). En este tipo de contextos sintácticos, la analogía resulta fundamental para el establecimiento de la estructura perifrástica progresiva. Así, en consonancia con las propuestas de Nickel (1967, 263), deben considerarse tres tipos fundamentales de estructuras que resultan muy similares:

1) Estructuras adjetivas atributivas: hie wæron lifiende ('ellos estaban vivos').

2) Participios apositivos: he wæs on alhne bewitende ('él estaba en el santuario, gobernando').

3) Y predicados nominales: hie wæron læfende credan monna ('ellos eran traidores de los hombres de fe').

Por tanto, es probable que estas formas sufrieran un proceso analógico que impulsó la constitución de la estructura progresiva verbal del IA. En el primero de los casos anteriores, el adjetivo atributivo en -ende puede reinterpretarse con facilidad como parte del complejo verbal. Este valor atributivo del participio presente en el ejemplo 1) wæron lifiende ('estaban vivos') produce la analogía sintáctica entre esta construcción con verbo cópula y adjetivo y la estructura progresiva wæron lifiende ('estaban viviendo'). En el segundo caso, ocurre un proceso analógico similar, pues la estructura apositiva de participio puede reinterpretarse como parte del paradigma verbal. Así, el ejemplo 2) he wæs on alhne bewitende ('él estaba en el santuario, gobernando') es equivalente a una estructura apositiva en el tiempo verbal simple: he wæs ('él estaba') + he bewiste ('él gobernaba'). Como puede apreciarse, la presencia del adjunto preposicional locativo en 2) on alhne ('en el santuario') favorece esta última 
interpretación. De esta forma, estructuras del tipo pa he wæs on alhne bewitende ('cuando él estaba en el santuario, gobernando'/ 'cuando él estaba gobernando en el santuario') posibilitan la analogía entre los participios apositivos y las construcciones progresivas de carácter verbal. Además, el patrón en 2) he wæs on alhne bewitende ('él estaba en el santuario, gobernando') guarda relación con un proceso de cambio semántico-pragmático en el que se evoluciona de una función descriptiva locativa, propia de la estructura apositiva, a un uso de tipo verbal, es decir, típicamente procesal de carácter aspectual. 4 En el tercer caso, las formas nominales de plural con el sufijo -ende; en otras palabras, las estructuras nomina agentis como el ejemplo en 3) læfende ('traidores'), pueden integrarse en el complejo verbal mediante el mismo fenómeno de analogía. En este último subtipo, la analogía se produce por la coincidencia en la flexión de caso genitivo entre la forma plural propiamente nominal wæron læfende ('eran traidores'), que gobierna la estructura de genitivo credan monna ('de los hombres de fe'), y la forma en participio presente wæron læfende credan monna ('estaban traicionando a los hombres de fe'). Esta ambigüedad, como señala Nickel (1966, 272), pudo dar lugar a la transición construccional siguiente:

$$
\begin{aligned}
& \text { be + nomina agentis + sintagma nominal (caso genitivo) > } \\
& \text { be + participio presente + sintagma nominal (caso genitivo/acusativo) }
\end{aligned}
$$

En favor de esta última interpretación, en IA un verbo en la forma simple tendía a gobernar un sintagma nominal en caso acusativo, mientras que la forma progresiva solía regir un sintagma nominal en caso genitivo. Esta transformación construccional cobra especial relevancia si se repara en los tipos verbales que admitían complementos tanto en caso genitivo como acusativo, pues hacia finales del IA las construcciones progresivas gobiernan mayoritariamente sintagmas nominales en caso acusativo.

De acuerdo con este último análisis, el carácter nominal de la construcción en IA resulta determinante para Traugott (1992, 189-190) en el establecimiento de la forma progresiva de tipo verbal. Según esta autora, en la línea de las propuestas de Dal (1952, 101-102), los contextos sintácticos preposicionales seguidos de un nominal, que permiten la analogía entre las formas de participio en -ing y los nominales en -ung, se registran ya durante el período del IA: pa he wæs on sawlunga ('cuando él estaba expirando'). No obstante, como explico en el apartado correspondiente, considero que este tipo de patrones preposicionales resulta más influyente en los desarrollos que experimenta la construcción en IM.

En cualquier caso, puede afirmarse que la construcción progresiva del IA consta de las estructuras y usos principales atestiguados en el período del IC, exceptuando las formas perfectas y pasivas conjugadas que surgen paralelamente en IM con el establecimiento del

4 Este tipo de procesos se ve favorecido en contextos en los que un verbo de la categoría semántica actividad presenta un mayor poder descriptivo en la forma de participio presente para denotar la acción de la que el sujeto de la oración es típicamente un agente ( $c f$. Goldberg, 1995; Langacker, 1991; Talmy, 2000, quienes describen procesos similares de alternancia locativa en construcciones de estructura argumental). 
complejo auxiliar verbal ( $c f$. Van Arkel-Simón, 2019). Como se verá al explicar la evolución de la construcción en IM, el creciente carácter analítico de la lengua se relaciona de manera señalada con el establecimiento de la construcción progresiva como marcador aspectual en inglés ( $c f$. Fisher, 1992, 250-256).

De este modo, en el plano gramatical, la construcción del IA comprende formas nominales típicamente deverbales, patrones propiamente verbales, y estructuras adjetivales. Estas últimas estructuras constituyen formas intermedias entre los dos primeros subtipos. En las formas verbales el auxiliar beon ('ser') por lo general denota un evento o la repetición de una actividad, y frecuentemente expresa también un tiempo futuro ( $c f$. Traugott, 1972, 90). Por su parte, el auxiliar wesan ('ser'/'estar') denota generalmente un estado que perdura en el tiempo del habla o que expresa la noción de habitualidad ( $c f$. Mustanoja, 1960, 583). Por su parte, el auxiliar weorðan ('convertirse') muestra una incidencia y una alternancia menores con respecto a las formas auxiliares antedichas, y denota habitualmente un origen, un cambio de estado, o la expresión de un tiempo futuro ( $c f$. Denison, 1993, 90). Dada su propia semántica, los dos primeros auxiliares favorecen la concepción de un proceso cuya acción es simultánea con el momento del habla, lo cual pudo también facilitar, como indica Traugott (1972, 90), el establecimiento del uso aspectual de la construcción. Esta última circunstancia, unida a la mayor carga semántica que conlleva el verbo weorðan ('convertirse'), parece explicar la desaparición de este último auxiliar en las formas progresivas verbales hacia finales del período del IA (Scheffer, 1975, 225). Seguidamente, en los ejemplos (3), (4) y (5) se ilustra respectivamente una ocurrencia prototípica de la estructura progresiva verbal, adjetival y nominal del IA: 5

(3) he on wisdome wel +deonde w+as

[he] [from] [wisdom] [well] [sucking] [was]

él, de la sabiduría, estaba aspirando bien

(cocathom2,+ACHom_II,_11:92.5.1867)

(4) syndon geliff +aste ealle lybbende gesceaftu

[are] [quickened] [all] [living] [creatures] todas las criaturas vivientes son vigorizadas

(ID coaelhom,+AHom_4:150.605)

(5) and hine m+arsode, his mihte herigende

[and] [him] [seethed] [his] [power] [(as a) flatterer]

y lo enfurecía, su poder como adulador

(coaelhom,+AHom_2:287.393)

5 Estos prototipos han sido extraídos del género de las Homilías, según mi propuesta de reclasificación de los géneros textuales para el conjunto York-Toronto-Helsinki-Penn (cf. Van ArkelSimón, 2018). 
Estructuralmente, el prototipo verbal más habitual del IA se construye con el verbo auxiliar en tiempo pasado como (3), y es infrecuente su concurrencia con formas pasivas o perfectas. En IA también es común su uso para establecer un marco de referencia para otra actividad, la cual es narrada en un contexto discursivo más amplio. En este último caso, habitualmente se emplea una frase de participio que carece de verbo auxiliar: ac us secga $+d$ lareowas $+t+$ at of fearres $f l+$ asce, fule stincende... ('pero los predicadores nos declaran que de la carne de la bestia, vilmente exhalando...'). ${ }^{6}$ Del mismo modo que ocurre en IC, la construcción perifrástica se emplea en este ejemplo prototípico (3) de la forma progresiva verbal del IA para indicar que la situación denotada por el verbo léxico está en progreso: +deonde $w+a s$ ('estaba aspirando'). Como sucede en este prototipo, la estructura progresiva del IA es mayoritaria en verbos que expresan una actividad como +deonde ('aspirando'), aunque en este período la construcción es también frecuente en verbos de carácter estativo. Probablemente, esta última circunstancia se vio favorecida por la persistencia de los subtipos adjetivales en los que el participio tiende a construirse a partir de verbos de carácter imperfectivo como (4) lybbende gesceaftu ('criaturas vivientes') (cf. Martín-Arista y Ojanguren-López, 2018).

Desde el punto de vista funcional, según las propuestas de Åkerlund (1911, 11-101), la forma verbal del IA consta de todos los usos descritos en IC, es decir, presenta usos aspectuales y no aspectuales ( $c f$. Núñez-Pertejo, 2004, 21-38; Smitterberg, 2005, 11-13; Kranich, 2010, 44-72; van Arkel-Simón, 2019). Así, en el ejemplo (3) he on wisdome wel +deonde $w+a s$ ('él, de la sabiduría, estaba aspirando bien') se aprecian todos los rasgos propios del uso aspectual: dinamismo, duración limitada, temporalidad y no completitud ( $c f$. Quirk et al., 1985, 198; Biber et al., 1999, 470-474; Huddleston y Pullum, 2002, 162-172).

Asimismo, los tipos progresivos verbales, nominales y adjetivales son habituales en IA, pues en el York-Toronto-Helsinki Parsed Corpus of Old English Prose (YCOE) se registran en total 6.025 ocurrencias progresivas. En cuanto a los tipos léxicos progresivos, en IA destacan los participios formados a partir de verbos que expresan movimiento, acción o repetición: faran ('ir'/ 'viajar'), gangan ('ir'), irnan ('correr'), feohtan ('luchar'), hergian ('saquear'), slean ('asesinar'), bærnan ('incendiar'), hangan ('colgar'), scinan ('brillar'), feolan ('persistir'), dreogan ('sobrellevar'), etc. Las formas progresivas se registran también en verbos que expresan un estado concebido en su extensión temporal o como un hábito al codificarse en la forma progresiva: libban ('vivir'), wunian ('habitar'), sorgian ('afligir'), winnan ('padecer'), etc. Además, el participio presente en IA se construye a partir de verbos de pensamiento, percepción o habla: cweban ('decir'), sprecan ('hablar'), gefeon ('regocijarse'), gemunan ('recordar'), gnornian ('lamentar'), etc.

Esta descripción de la naturaleza de la construcción progresiva en IA parece indicativa de la persistencia de estas formas en inglés desde sus primeros períodos. Sin embargo, la importancia de cada una de las variantes estructurales y funcionales presenta patrones

5 Ejemplo extraído del género de las Homilías del York-Toronto-Helsinki Parsed Corpus of Old English Prose (YCOE): ID coaelhom,+AHom_1:267.139. 
distintivos en IA. En primer término, la influencia de las formas latinas de participio, los predicados nominales y los patrones adjetivales atributivos y apositivos del IA parecen contribuir a la constitución de la construcción mediante un proceso de analogía.

Asimismo, aunque la función aspectual es la mayoritaria en las formas verbales de la construcción, pues esta denota principalmente una duración implícita y un aspecto imperfectivo, se trata de una estructura aún no obligatoria para codificar este tipo concreto de especificación aspectual. En muchos casos, el uso de la construcción en IA se vincula con el aporte de una descripción más vívida o emotiva de la situación contenida en la proposición, lo que se relaciona con los usos no aspectuales que la caracterizan de manera destacada durante los períodos medievales ( $c f$. Goedsche, 1932, 475; Mustanoja, 1960, 585). Así, las formas progresivas del IA aparentan constituir un recurso estilístico que, en un primer momento, probablemente encuentra inspiración en las glosas de los textos latinos. En este sentido, en todos los casos, la alternancia de la construcción con la forma no progresiva no aparenta implicar un cambio sustancial en el contenido de la proposición más allá de expresar contenido emocional o de cumplir funciones de tipo descriptivo o estilístico ( $c f$. Mitchell, 1976, 484-485; 1985, §685; Nickel, 1966, 267; Nehls, 1988, 180).

Por su parte, la relativa variedad que presentan los tipos léxicos progresivos del IA parece indicativa de la existencia de un proceso de gramaticalización primario, así como de fenómenos de cambio construccional ( $c f$. Kranich, 2010).7 En mi opinión, este tipo de cambios son concomitantes con la desaparición del sistema de afijación verbal para codificar la categoría aspectual perfectiva del inglés ( $c f$. Traugott, 1990, 500; Traugott, 2003, 71-98; Fisher, 2007, 259). Por tanto, aunque la forma progresiva verbal cumple la función de codificar la categoría aspectual imperfectiva, así como denotar la noción implícita de duración, esta no constituye aún el patrón gramatical obligatorio para ello.

\subsection{La construcción progresiva en inglés medio}

La primera circunstancia reseñable en el desarrollo de la construcción progresiva en IM es su descenso en términos absolutos con respecto al período anterior. Más concretamente, se registran 3.843 formas progresivas totales en el Penn Parsed Corpus of Middle English (PPCME2). Sin embargo, a pesar de este descenso en las ocurrencias de la construcción durante el IM, existe una evolución continuada y no fragmentada o vicaria de la misma, pues en IM se registran todos los usos y estructuras atestiguados durante el período anterior. En defensa de esta tesis, el descenso en las ocurrencias progresivas totales y verbales en IM no se

\footnotetext{
7 En la gramaticalización primaria, las estructuras que la experimentan adquieren carácter de construcción sintáctica. En la gramaticalización secundaria, las construcciones presentan un significado gramatical pleno. La subjetivación conlleva la ampliación de los significados de la construcción para expresar la percepción del emisor. En la objetivación, una vez que el uso de la construcción se hace obligatorio en determinados contextos sintácticos, esta pierde su capacidad de ampliar los significados subjetivos.
} 
corresponde con una disminución de su carga semántica, ni tampoco con una merma en los patrones estructurales que caracterizan a la construcción.

Con todo, la naturaleza de esta estructura perifrástica en IM puede también interpretarse considerando los procesos generales de cambio lingüístico en los que está inmersa la lengua durante este período.

En primera instancia, en IM existe una considerable expansión de las variantes perifrásticas que tiene lugar en virtud de la constitución del sistema verbal auxiliar. Las formas perfectas y modales y el auxiliar do comienzan a establecerse en este período para producir los patrones sintácticos que serán característicos del inglés moderno. Así, el desarrollo del sistema auxiliar verbal es concomitante con la pérdida del sistema de casos del IA, pues hacia finales de este último período no solo se registra la casi total desaparición del sistema de oposiciones mediante prefijación verbal, sino que además se reduce drásticamente el sistema gramatical de caso para los argumentos verbales. En IM este sistema se restringe ya al caso nominativo para codificar la función sintáctica de sujeto y al caso oblicuo para expresar las funciones sintácticas de objeto en los complementos verbales.

En función de este proceso, hacia finales del IM la distinción morfológica entre el caso nominativo y el oblicuo es ya prácticamente inexistente en los sustantivos, y tan solo se mantiene de forma distintiva en los pronombres personales. Esta dramática transformación del sistema gramatical del inglés, que abandona su carácter sintético para dar lugar a un sistema de corte analítico, es fundamental en el desarrollo de las estructuras perifrásticas progresivas. En IM se registran estructuras progresivas perfectas, pluscuamperfectas y futuras, aunque aún son infrecuentes las formas progresivas en construcciones pasivas y futuras perfectas ( $c f$. Fisher, 1992, 233-256). Asimismo, durante este período se registra la casi total desaparición del auxiliar worthen ('convertirse'), ya que, al tener lugar la constitución del sistema auxiliar verbal, su valor semántico intrínseco le impide competir con las formas del verbo ben ('ser/ estar'). Como resulta lógico, este nuevo sistema auxiliar requería de unos lexemas con una carga semántica proclive a ser neutra y de una menor complejidad fonética y morfológica.

Por su parte, la distinción entre los participios presentes y los sustantivos de carácter verbal también se desvanece durante el IM. Así, la sufijación en -ende y las variantes morfológicas propias del participio presente -and, -ande e -inde experimentan un proceso de fusión con el sufijo -ung y las variantes morfológicas características de los sustantivos de carácter verbal -yng, -ynge e -inge. Esta fusión da lugar a la forma indistinta -ing que ha perdurado hasta nuestros días, la cual representa ambas categorías gramaticales desde finales de este período. En estos procesos de aglutinación y reducción fonética, que dan lugar a la forma en -ing, resulta especialmente relevante la analogía que se establece entre la forma de participio presente en -inde y la estructura nominal en -inge.

Estos procesos de coalescencia y reanálisis morfológico que comienzan en IM motivan el establecimiento de la categoría gramatical de gerundio tal y como la conocemos en el inglés actual ( $c f$. Lass, 1992, 144-146). En estos contextos de reanálisis morfológico, los patrones de 
participio en estructuras preposicionales locativas cobran relevancia en el desarrollo de la construcción progresiva del IM: ${ }^{8}$

(6) beast $+t t$ is to reachinde Abuten

[beast] [that] [is] [into] [wandering] [about]

animal que está de merodeo

(CMANCRIW-1,II.113.1407)

Por su parte, los tipos nominales preposicionales en -ing, que se derivan del sufijo en -ung del IA, devienen en un proceso de reanálisis con estructuras como las inmediatamente anteriores:

(7) thy wille in kepynge this maydenhode

[your] [will] [in] [caring about] [this] [celibacy]

tu voluntad de guardar este celibato

(CMAELR4,10.266)

Esto dará lugar a los patrones verbales preposicionales locativos en -ing:

(8) is ine knauyngewy+t-oute wy+tnimynge

[is] [inside] [knowing] [outside][misfortune]

está en (el proceso de) enterarse sin desdicha

(CMAYENBI,82.1606)

Sin embargo, estos tres tipos de estructuras coexisten con las formas verbales, adjetivales y nominales de la construcción progresiva, que continúan con su desarrollo directo a partir de los patrones del IA:

(9) Tus beo+dgederinde ancres of god

[thus] [is] [assembling] [weight] [from] [God]

por tanto, está armándose de la fuerza de Dios

(CMANCRIW-1,II.103.1263)

(10) ha is grucinde \& dangerus

[he] [is] [overbearing] [and] [haughty]

él es autoritario y arrogante

(CMANCRIW-1,II.85.1032)

${ }^{8}$ Los prototipos de esta sección han sido extraídos del género de los Tratados según mi propuesta de reclasificación de los géneros textuales del conjunto York-Toronto-Helsinki-Penn. 
(11) +ge nebeon nautilich the scheunchinde hors

[they] [were not] [not] [same] [the] [shyness] [horse]

ellos no tenían la misma timidez que el caballo

(CMANCRIW-1,II.178.2495)

Así, las estructuras preposicionales locativas del tipo (6) beast + tt is to reachinde Abuten ('animal que está de merodeo') y (8) is ine knauynge wy+t-oute wy+tnimynge ['está en (el proceso de) enterarse sin desdicha'] favorecen la analogía semántica con los significados de duración implícita y carácter imperfectivo característicos de la construcción aspectual en (10): Tus beo+d gederinde ancres of god ('por tanto, está armándose de la fuerza de Dios'). Esta analogía semántica facilita los procesos de reanálisis morfológico entre las formas derivadas de los tipos nominales preposicionales en -ung y las descendientes de los participios presentes en -inde, lo que conduce al sufijo -ing que se empleará finalmente en todos los casos. En este sentido, el proceso de asimilación entre las formas nominales preposicionales como (8) y los participios presentes como (9) tuvo lugar en un período en el que la necesidad de emplear preposiciones para expresar relaciones sintácticas adquiría especial relevancia, dada la pérdida del sistema gramatical de caso durante el inglés medio. De esta manera, este nuevo sistema de relaciones analíticas ocasionó diversos procesos de reanálisis sintáctico que también facilitaron la analogía entre los dos tipos de construcciones anteriormente mencionadas: las formas nominales preposicionales y los participios presentes.

En estos contextos gramaticales, durante el IM el sustrato céltico existente en Gran Bretaña pudo favorecer los procesos de reanálisis sintáctico y analogía morfológico-semántica antedichos. Como se ha indicado anteriormente, los patrones preposicionales nominales de carácter verbal parecen ser frecuentes en los dialectos celtas antiguos y también medios, puesto que, en primer término, estas lenguas carecen de formas de infinitivo. Asimismo, las estructuras perifrásticas aparentan ser habituales en estos dialectos célticos ( $c f$. NúñezPertejo, 2004, 120). Así, del mismo modo que ocurre en el período anterior, en IM los desarrollos de la construcción progresiva se imbrican indefectiblemente con los procesos generales de evolución de la lengua.

En cualquier caso, como puede extraerse de los ejemplos (6) a (11), la construcción progresiva del IM consta de todos los usos registrados en IA y su naturaleza es muy similar a la del período anterior. En síntesis, la construcción presenta mayoritariamente usos aspectuales y no aspectuales en las formas verbales, así como no aspectuales en las estructuras de tipo adjetival y nominal. En lo que respecta a las funciones aspectuales de las formas verbales, durante el período del IM se constata también la expresión de dinamismo, duración limitada, temporalidad y no completitud. En relación con los usos no aspectuales, se aprecia la expresión de subjetividad, ya sea esta mediante especificación adverbial o por medio de un uso descriptivo.

Sin embargo, el uso de la construcción para denotar la función aspectual aún no es obligatoria en este período. De este modo, también en IM, el uso de esta parece identificarse fundamentalmente con un propósito descriptivo o con la expresión de contenido emocional, 
por lo que aún representa una mera variante estilística de las formas en los tiempos verbales simples. No obstante, la construcción verbal continúa denotando en IM aspecto imperfectivo y duración implícita, lo que resulta también indicativo de la continuidad de estas estructuras con respecto al período anterior ( $c f$. Scheffer, 1975, 218-249).

Los tipos léxicos a partir de los que se construyen las estructuras progresivas en IM incluyen también verbos de movimiento o que pueden conceptualizarse como una actividad: fighten ('luchar'), gă̄ngen ('ir'), brennen ('arder'), bernen ('almacenar'), cǒmen ('llegar'), gōn ('caminar'), sẹcchen ('buscar'), rennen ('correr'), dwĕlen ('deambular'), enquēren ('investigar'), waxen ('desarrollarse'), abìden ('esperar'), etc. Son también frecuentes los verbos estativos que pueden concebirse en una extensión temporal o que denotan habitualidad: flouen ('fluir'), slẹpen ('dormir'), joinen ('conectar'), consenten ('convenir'), lasten ('continuar'), wǒnen ('residir'), liven ('habitar'), lŏngen ('languidecer'), etc. Asimismo, la construcción es habitual con verbos que denotan estados de pensamiento, mentales y situaciones del habla: drēden ('temer'), thinken ('pensar'), spēken ('hablar'), etc.

Esta relativa variedad de tipos léxicos progresivos, la cual aumenta con respecto al período anterior, en combinación con los procesos de reanálisis morfológico y sintáctico anteriormente descritos, parece indicativa de que nos encontramos ante un período en el que existen fenómenos de gramaticalización primaria y cambio construccional.

Estos fenómenos se identifican, fundamentalmente, por la subjetividad que confiere el uso de los diferentes subtipos progresivos. Así, en el ejemplo (9) del IM Tus beo+d gederinde ancres of god ('por tanto, está armándose de la fuerza de Dios') puede apreciarse una cierta subjetivación de la estructura en la que se inserta la construcción progresiva con respecto al prototipo verbal del IA, la cual se ilustra en la ocurrencia (3) del apartado anterior he on wisdome wel +deonde w+as ('él, de la sabiduría, estaba aspirando bien'). Esta subjetivación se atestigua en el distanciamiento por parte del hablante con respecto a la situación denotada por el verbo en la forma progresiva.

En cualquier caso, la prominencia de las funciones especiales y no aspectuales propias de los participios presentes que se atestiguan en los períodos medievales constituye un argumento en defensa de la existencia de este fenómeno de subjetivación que caracteriza los procesos de gramaticalización primaria. En este tipo de transiciones gramaticales, se produce la desemantización de la forma verbal de participio, pues esta resulta en una estructura con menor carga semántica que la existente en el período anterior.

En este tipo de procesos, la pérdida de capacidad de proyección sintáctica del participio presente es un resultado del reajuste gramatical que experimenta la construcción durante el IM, lo que produce una progresiva fijación de las posiciones sintácticas que ocupa la estructura perifrástica. En este sentido, el fenómeno de reanálisis morfológico en que devienen las formas de participio, que da lugar a la erosión fonética de los diferentes sufijos para constituir la terminación en -ing, señala la progresiva pérdida de propiedades morfosintácticas de la construcción en el tránsito del IA al IM. 
Esta aglutinación de las formas de participio presente es también paralela al aumento del rango paradigmático de la construcción, puesto que, al constituirse la categoría auxiliar verbal, se aprecia cómo la construcción comienza a combinarse de manera complementaria con el resto de las estructuras auxiliares para conjugar las formas perfectas, pluscuamperfectas y futuras que se atestiguan desde el IM. Por tanto, la construcción progresiva comienza a condensarse y cohesionarse con el resto de los miembros de la categoría auxiliar durante este período.

Este conjunto de parámetros principales viene a definir el carácter primario de los procesos de gramaticalización que experimentan las formas progresivas durante los períodos medievales, el cual, según mi valoración, es concomitante con diversos procesos de cambio construccional. En este sentido, las transiciones semántico-pragmáticas y estructurales de las diferentes formas de participio que ocurren en estos primeros períodos del inglés son fruto del paulatino proceso de constitución de la construcción como categoría gramatical que expresará de manera obligatoria la noción aspectual. Así, en estos dos primeros apartados sobre la evolución de las construcciones progresivas he descrito cómo las configuraciones gramaticales de las formas nominales y adjetivales contribuyen al desarrollo del valor aspectual de las formas típicamente verbales que será prominente en los períodos modernos.

Por tanto, este tipo de cambios a nivel microconstruccional se corresponde con la paulatina semantización de las estructuras progresivas como unidades especializadas de significado, ya que, a nivel paradigmático, se está reconfigurando todo el sistema gramatical de la lengua. En el nuevo sistema analítico del inglés, la reconfiguración sintáctica en que devienen las microconstrucciones progresivas generará finalmente una macroconstrucción obligatoria para codificar la categoría aspectual durante los períodos modernos de la lengua.

\subsection{El desarrollo de la construcción progresiva en inglés moderno temprano}

El valor primario como marcador aspectual de la construcción progresiva se constituye durante el IMT cuando se convierte en la categoría gramatical obligatoria para codificar esta función. De este modo, durante este período se registran todas las formas verbales conjugadas en tiempo, modo y voz de la construcción ( $c f$. Rissanen, 1999, 216-218).

El subtipo verbal de la construcción se convierte definitivamente en la estructura más importante de las variantes estructurales durante el IMT, pues sus funciones aspectuales características conllevan la gradual desaparición de los patrones preposicionales de carácter nominal. Este último desarrollo coincide, por su parte, con el establecimiento de las formas de gerundio modernas. La dramática expansión de todas las formas de la construcción durante el IMT queda avalada por el número total de ocurrencias progresivas registradas en el Penn-Helsinki Parsed Corpus of Early Modern English (PPCEME), que contiene un total de 18.946 de estas estructuras.

Este aumento exponencial en las frecuencias de aparición de las formas progresivas se debe principalmente a la nueva posición que ocupa la construcción en el sistema gramatical de la lengua. Así, las estructuras progresivas del IMT constan de los patrones verbales, 
adjetivales y nominales que se registran desde el IA, pero se extienden en todo tipo de contextos lingüísticos: ${ }^{9}$

1) Formas verbales:

(12) $w=c h=i s$ only becomeing to herself (ALHATTON-E3-H,2,241.17)

(13) Mr Speaker was coming through Westminster Hall (AUNGIER-E3-H,25,P.16)

(14) but will not be failling to doe (ANHATTON-E3-P1,2,154.22)

(15) thou shalt liveing or dying (HOXINDEN-1640-E3-P1,114.117)

(16) their custome has ever ben going on violently (EVELYN-E3-H,904.166)

(17) and he had been preparing his Notes (BURNETROC-E3-H,20.98)

(18) the Grace to be working out my Salvation (EVELYN-E3-P1,914.273)

(19) which being done long ago (BURNETROC-E3-P2,88.27)

2) Formas adjetivales:

(20) his Conversation was easie and obliging (BURNETROC-E3-H,7.25)

(21) their professing and swearing Friendship (BURNETROC-E3-H,24.112)

3) Formas nominales:

(22) with the Cardinall's retrenching my pention (CHARLES-1650-E3-P1,10,B.37)

(23) that my being about him could have been of any use (BURNETROC-E3-H,137.135)

Esta amplia diversidad estructural se debe a la expansión paradigmática en que deviene la construcción durante el inglés moderno temprano. De este modo, las formas verbales en tiempo presente y pasado, como los ejemplos (12) y (13), continúan siendo las mayoritarias en el PPCEME. Le siguen, respectivamente, las formas perfectas como (16) y (17), las futuras como (14), y las formas de infinitivo como (18). Las estructuras modales como el ejemplo en (16) son las menos frecuentes. Por último, la estructura pasiva (19) which being done long ago comienza a sustituir en este período a la estructura apositiva de participio doble en voz activa, que se empleaba para denotar un significado pasivo:

(24) the force of these motions, being, for most part, according to the Impression that was made on our brains (BURNETROC-E3-P1,49.96)

Como se verá en el apartado siguiente, en IModT se completará la conjugación de la forma progresiva en voz pasiva derivada de los patrones en (19). En este proceso, el fenómeno de reanálisis sintáctico de la forma conjugada del verbo be como auxiliar, que se inicia en IM, ayudará al establecimiento de la forma pasiva moderna. Así, las formas no personales del auxiliar be se interpretarán como verbos léxicos, mientras que las formas conjugadas se considerarán parte de la categoría auxiliar. Asimismo, desde la perspectiva funcional, el

9 Los ejemplos de esta sección han sido extraídos del género de la Biografía según mi propuesta de reclasificación de los géneros textuales del conjunto York-Toronto-Helsinki-Penn. 
carácter obligatorio que adquiere la construcción para codificar la categoría aspectual en IMT contribuye al establecimiento de las estructuras pasivas conjugadas, las cuales completarán prácticamente todo el paradigma progresivo actual en IModT ( $c f$. Kranich, 2010, 119-128). Además, como puede apreciarse en los prototipos extraídos, las formas progresivas adjetivales continúan su desarrollo directo desde el período medieval, registrándose funciones atributivas como (20) his Conversation was easie and obliging, y modificadoras como (21) their professing and swearing Friendship.

Por su parte, la categoría actual de gerundio se establece de manera casi definitiva en IMT, pues comienzan a ser frecuentes las estructuras progresivas de genitivo como el ejemplo (22) with the Cardinall's retrenching my pention. Estos patrones de gerundio, de carácter típicamente nominal, supondrán una extensión de las estructuras de participio presente nominales del tipo (23) that my being about him could have been of any use, las cuales continúan también una evolución directa a partir de las formas medievales. Con todo, la drástica expansión estructural del paradigma progresivo queda reflejada por el gran número de construcciones que contienen formas de participio en el PPCEME, más específicamente:

1) Estructuras apositivas de participio presente: I recomend me to your ladyship, evermore desiring to wit (ABOTT-E1-P1,229.4)

2) Patrones preposicionales: Lady North was conserned in making $y=e=$ (ALHATTON2-E3-P1,64.36)

3) Estructuras existenciales: there being nothing to be liked in him (ALHATTON-E3$\mathrm{H}, 2,241.7)$

4) Y formas elípticas sin auxiliar: an Armie beinge alreadie put to the Seas (ELIZ-1580E2-P1,2.3,139.54)

Estas variantes estructurales, que resultan numerosas, generalmente representan oraciones o frases de participio que cumplen una función circunstancial, o bien se trata de formas seudoprogresivas inmersas en el proceso de constitución de la categoría verbal propia mediante analogía.

Funcionalmente, en IMT se atestigua también la prominencia de los usos progresivos que son característicos del IC. Entre ellos, los usos aspectuales que implican la expresión de dinamismo, duración limitada, temporalidad y no completitud son los más importantes, y comienzan a adquirir carácter obligatorio en este período para codificar la categoría aspectual progresiva. El patrón léxico-gramatical final que presentan estos componentes resulta de la expansión paradigmática que experimenta la construcción en IMT, ya que la fijación de las posiciones sintácticas del auxiliar be y de su complemento sintagmático verbal de participio se produce en virtud de la obligatoriedad que adquiere la estructura en el sistema gramatical del IMT para codificar el tipo aspectual progresivo.

La consolidación de este carácter obligatorio se explica en virtud de los procesos de gramaticalización secundaria y construccionalización que experimenta la construcción durante los períodos modernos de la lengua. Estos procesos permitirán que la construcción cumpla de manera preponderante las funciones aspectuales primarias que la definen en el 
sistema gramatical del inglés actual ( $c f$. Kranich, 2010). Con todo, las funciones que confieren subjetividad a la proposición en la que se inserta la forma progresiva verbal experimentan una disminución en IMT. La expansión paradigmática de los patrones estructurales, propia de los períodos modernos, conlleva la objetivación de las estructuras en las que aparecen las formas progresivas, pues estas presentan carácter obligatorio para expresar el aspecto, lo que sustrae otras denotaciones semántico-pragmáticas. Esta objetivación se aprecia en el acercamiento a la perspectiva del hablante con respecto a la situación denotada por el verbo en la forma progresiva.

Por último, los tipos semánticos verbales a partir de los que se construyen las formas progresivas experimentan también una clara expansión en IMT. Así, continúan siendo mayoritarios los verbos de movimiento o que pueden conceptualizarse como una actividad: go, make, do, come, prepare, smile, serve, bite, shew, pass, wait, fall, begin, direct, take, unite, etc. Por su parte, las formas progresivas en verbos estativos son menos frecuentes que en los períodos medievales: live, confirm, love, grow, inhabit, dye, stand, agree, fade, owe, remain, etc. Asimismo, se registran estructuras progresivas en verbos de pensamiento, estados mentales, percepción y habla: imagin, see, conceive, satisfy, doubt, remember, wander, desire, judge, know, think, say, talk, etc.

\subsection{La consolidación de la construcción progresiva en inglés moderno tardío}

La construcción progresiva completa su proceso de gramaticalización en IModT. Al consolidarse su función gramatical, la paulatina fijación de los contextos paradigmáticos en los que alterna en régimen de complementariedad con otras construcciones léxicogramaticales limitará las posibilidades de enriquecimiento semántico-pragmático que caracterizan los usos subjetivos de la construcción. De este modo, la asociación obligatoria de la construcción con la función primaria de marcador aspectual se consuma en IModT, e impide muchos de los contextos propios de los períodos medievales en los que la forma progresiva se emplea como recurso expresivo. De esta forma, la complejidad semánticopragmática de la perífrasis aspectual y su capacidad de cumplir funciones expresivas disminuyen notablemente durante el IModT ( $c f$. Kranich, 2010, 245). Este proceso de objetivación funcional y la obligatoriedad que adquiere la construcción para indicar aspecto progresivo definen el carácter secundario del fenómeno de gramaticalización que experimenta la construcción en los períodos modernos ( $c f$. Kranich, 2010; Petré, 2015).

En este período, los tipos léxicos progresivos comprenden principalmente verbos de movimiento o que pueden ser conceptualizados como una actividad: do, try, form, sit, lose, multiply, treat, go, rest, soar, make, undergo, shift, revolve, seek, happen, allow, dispose, give, take, put, etc. Se registran también verbos estativos que pueden interpretarse en una extensión temporal o como un hábito: live, last, win, deserve, remain, engage, perish, owe, etc. Asimismo, la construcción continúa siendo frecuente en verbos de pensamiento, habla o percepción: expect, desire, perceive, know, think, reason, understand, speak, see, look, etc. $\mathrm{El}$ aumento exponencial que experimentan las formas progresivas se mantiene en IModT, 
registrándose una frecuencia de aparición de 14.284 ocurrencias totales en el Penn-Helsinki Parsed Corpus of Modern British English (PPCMBE). Esta tendencia se relaciona de forma directa con los procesos de gramaticalización secundaria y cambio construccional que comienzan desde el período anterior.

De este modo, una vez que el valor gramatical obligatorio de la macroconstrucción verbal se asienta, el uso de la misma resulta necesario en un número mayor de contextos lingüísticos. En este entorno de cambio gramatical, destaca la consolidación de la estructura progresiva al constituirse definitivamente la forma pasiva verbal durante el IModT. Este último desarrollo se produce cuando las posibilidades de alternancia morfosintáctica del complejo auxiliar verbal se ven asimismo reducidas, pues el esquema de construcción de la categoría auxiliar del inglés, tal y como ha perdurado hasta nuestros días, se establece plenamente durante este período. De esta manera, la constitución del sistema auxiliar verbal favorece el establecimiento de las formas progresivas en los tipos pasivos. Así, el fenómeno de construccionalización que experimenta la forma progresiva en los períodos modernos podría asimilarse con el proceso de reanálisis sintáctico que experimenta el participio presente como modificador o complemento sintagmático del núcleo auxiliar verbal. Por tanto, en IModT se completa la pérdida del rol de participio presente como núcleo de su propio sintagma. En cualquier caso, las formas pasivas más habituales en IModT continúan construyéndose en los tiempos verbales presente y pasado simple (ibid.):

\section{(25) I am again being bombarded about Eton (BENSON-190X,107.53) \\ (26) she were being photographed (BENSON-190X,124.596)}

Con todo, la evolución estructural de las construcciones progresivas aparenta mostrar una continuidad en los patrones propiamente verbales, adjetivales y nominales que se registran desde el IA. Estos patrones del IModT se ejemplifican, respectivamente, en (27), (28) y (29). Asimismo, se mantiene una alta incidencia de las frases y oraciones de participio, que se ilustran en (30):

(27) I hope you are at this time sitting down (AUSTEN-180X,160.7)

(28) the good humour of his countenance is quite bewitching (AUSTEN-180X,161.24)

(29) My writing to you \$today prevents Eliz=th's= writing to Harriot (AUSTEN-180X,163.73)

(30) Fanny was met walking with Miss Sharp (AUSTEN-180X,161.38)

\section{Conclusiones}

El desarrollo diacrónico sostenido de los patrones estructurales de la forma progresiva se acompaña también de la continuidad en el contenido semántico-pragmático que conlleva la construcción, lo que parece configurar un gradiente estructural y funcional en las formas progresivas a lo largo de su evolución. Así, el concepto de gradiente lingüístico resulta 
particularmente adecuado para la explicación de los patrones gramaticales, los rasgos semántico-pragmáticos y los procesos de cambio gramatical que caracterizan a las construcciones progresivas inglesas a lo largo de toda su evolución.

Los cambios sutiles en este gradiente caracterizan a la construcción en los planos formal y semántico según el período, y asimismo posibilitan, en cada período, la identificación de los principales procesos de cambio gramatical en curso. En este sentido, los usos aspectuales y no aspectuales se registran desde el IA, pero la prominencia de cada uno de ellos varía a lo largo del tiempo en virtud de la función primaria que desempeña la estructura y la naturaleza del proceso de cambio gramatical que tiene lugar ( $c f$. 3.2. y 3.4.).

En IA, el uso de la construcción se relaciona con el aporte de una expresión más vívida de la situación descrita, por lo que cumple predominantemente propósitos estilísticos y emocionales.

En IM, las funciones estilísticas continúan siendo preponderantes, pero comienza a desarrollarse la función aspectual al tiempo que disminuyen los usos no aspectuales. En este contexto funcional característico del inglés medieval, se está asimismo reconfigurando morfosintácticamente el sistema gramatical de la lengua para constituir un nuevo ordenamiento de carácter analítico. De esta forma, en IM se aprecian fenómenos de gramaticalización primaria y cambio construccional, los cuales se encuentran con toda probabilidad en curso desde el período anterior. En apoyo de esta tesis, la reconfiguración gramatical que conllevó la pérdida del sistema aspectual de prefijación verbal se completa hacia finales del IA, lo que hizo necesario el desarrollo de una construcción aspectual alternativa. Por tanto, la subjetivación de la estructura es más acusada en IM, pues se potencian los usos no aspectuales de las formas verbales debido al enriquecimiento semántico-pragmático que conlleva el desarrollo de una nueva función construccional. En IA, aun existiendo los mismos usos, la expresión de subjetividad que confiere la construcción resulta menor (cf. 3.1. y 3.2.).

Por su parte, en IMT la obligatoriedad de la estructura para codificar aspecto progresivo comienza a perfilar el uso primario de la construcción. Esta obligatoriedad se consolida definitivamente durante el IModT. Este proceso determina que los usos aspectuales confieran objetividad al polo funcional propiamente aspectual de la estructura, y se registran también usos no aspectuales, los cuales caracterizan el polo funcional que expresa subjetividad durante el período moderno. De esta forma, los usos subjetivos representan una continuación de la expresión de subjetividad que es característica de la construcción desde su origen, pero coexisten ahora con el carácter primario de la función aspectual que desempeña la construcción. En este gradiente funcional, en todos los períodos la estructura progresiva expresa intrínsecamente contenido emocional o algún tipo de subjetividad. Por tanto, el valor semántico que conlleva la noción de una duración implícita y aspecto imperfectivo en la construcción verbal comienza en el período del IA, y deviene en una especialización gradual en el sistema gramatical de la lengua hasta adquirir carácter plenamente obligatorio para codificar la categoría en IMT e IModT. 
En este desarrollo de la construcción progresiva resultan fundamentales los procesos particulares de cambio gramatical, los cuales, como se ha mostrado, se enmarcan indefectiblemente en los contextos generales de cambio lingüístico que experimenta la lengua en cada período. Así, la paulatina pérdida del sistema de prefijación verbal para codificar aspecto perfectivo, así como la gradual obligatoriedad que adquiere la perífrasis verbal progresiva para expresar aspecto imperfectivo y una duración implícita, configuran los principales patrones de alternancia en las distinciones aspectuales de la lengua.

En este contexto, el concepto de gradiente lingüístico posibilita definir las características preponderantes de la estructura en cada período, ya que perfila la sucesiva alternancia de los rasgos funcionales, patrones estructurales y procesos de cambio gramatical prominentes, lo que da lugar a la caracterización de las relaciones que esta construcción establece con sus variantes paradigmáticas no aspectuales en el sistema gramatical de la lengua.

\section{REFERENCIAS BIBLIOGRÁFICAS}

ÅkERLund, A. 1911. On the History of the Definite Tenses in English. Lund: Berlingska Boktryckeriet.

BAUGH, A. C. y CABLE, T. 2002 (1935). A History of the English Language. London: Routledge. BIBER, D. 1988. Variation across Speech and Writing. Cambridge: Cambridge University Press. BiBER, D. y Finegan, E. 1997. "Diachronic Relations Among Speech-Based and Written Registers in English”, en Nevalainen, T. y Kahlas-Tarkka, L. (eds.), To Explain the Present. Studies in the Changing English Language in Honour of Matti Rissanen. Helsinki: Société Néophilologique, pp. 253-275.

Biber, D., Johansson, S., Leech, G., Conrad, S. y Finegan, E. 1999. Longman Grammar of Spoken and Written English. London: Longman.

DAL, I. 1952. "Zur Entstehung des Englischen Participium Praesentis auf -ing”, Norsk Tidsskrift for Sprogvidenskap, 16, pp. 5-116.

DENISON, D. 1993. English Historical Syntax: Verbal Constructions. New York: Longman.

Fisher, O. 1992. "Syntax", en Blake, N. (ed.), Vol. 2 of The Cambridge History of the English Language. Cambridge: Cambridge University Press, pp. 207-408.

FisHeR, O. 2007. Morphosyntactic Change. Functional and Formal Perspectives. Oxford: Oxford University Press.

GEISLER, C. 2002. "Investigating Register Variation in Nineteenth-Century English", en Reppen, R., Fitzmaurice, S. M. y Biber, D. (eds.), Using Corpora to Explore Linguistic Variation. Amsterdam/Philadelphia: John Benjamins, pp. 249-271.

Goedsche, C. R. 1932. "The Terminate Aspect of the Expanded Form: Its Development and Its Relation to the Gerund", The Journal of English and Germanic Philology, 31, pp. 469-477.

GoldBerg, A. E. 1995. Constructions: A Construction Grammar Approach to Argument Structure. Chicago: Chicago University Press.

HogG, R. y Denison, D. 2006. A History of the English Language. Cambridge: Cambridge University Press. 
Huddleston, R. y Pullum, G. K. 2002. The Cambridge Grammar of the English Language. Cambridge: Cambridge University Press.

JESPERSEN, O. 1931. A Modern English Grammar on Historical Principles. Heidelberg: Carl Winters Universitätsbuchhandlung.

KILLIE, K. 2014. "The Development of the English BE + V-ende/V-ing Periphrasis: from Emphatic to Progressive Marker?”, English Language and Linguistics, 18(3), pp. 361-386. https://doi.org/10.1017/S1360674314000148.

Kranich, S. 2010. The Progressive in Modern English: A Corpus-Based Study of Grammaticalization and Related Changes. Amsterdam/New York: Rodolpi.

Kroch, A. y TAYLOR, A. 2000. Penn-Helsinki Parsed Corpus of Middle English (PPCME2) (2nd ed.) [CD-ROM]. Philadelphia: University of Pennsylvania.

Kroch, A., Santorini, B. y Diertani, A. 2004. Penn-Helsinki Parsed Corpus of Early Modern English (PPCEME) (1st ed.) [CD-ROM]. Philadelphia: University of Pennsylvania.

Kroch, A., SANTORINI, B. y Diertani, A. 2010. The Penn Parsed Corpus of Modern British English (PPCMBE) (1st ed.) [CD-ROM. Philadelphia: University of Pennsylvania.

LANGACKER, R. W. 1991. Fundations of Cognitive Grammar: Descriptive Application. Stanford: Stanford University Press.

LASS, R. 1992. "Phonology and Morphology", en Blake, N. (ed.), Vol. 2 of The Cambridge History of the English Language. Cambridge: Cambridge University Press, pp. 23-154.

Martín-Arista, J. y OJANGURen-López, A. E. 2018. "The Adjectival Participle with bēon in Old English -. A Morpho-syntactic Analysis”, Selim, 23, pp. 27-53. Disponible en: https://reunido.uniovi.es/index.php/SELIM/article/view/13296/12031.

Mitchell, B. 1976. "Some Problems Involving Old English Periphrases with beon/wesan and the Present Participle”, Neuphilologische Mitteilungen, 77(4), pp. 478-491.

Mitchell, B. 1985. Old English Syntax. Vols. 1 y 2. Oxford: Oxford University Press.

Mitchel, B., y RoBInson, F. C. 2002. A Guide to Old English. Oxford/New York: Basil Blackwell.

Mossé, F. 1938. Histoire de la Forme Périphrastique être + participe présent en Germanique. Paris: C. Klincksiek.

Mustanoja, T. F. 1960. A Middle English Syntax. Part 1. Parts of Speech. Helsinki: Societé Néophilologique.

NEHLS, D. 1988. "On the Development of the Grammatical Category of Verbal Aspect in English”, en Klegraf, J. y Nehls, D. (eds.), Essays on the English Language and Applied Linguistics: On the Occasion of Gerhard Nickel's 6oth Birthday. Heidelberg: Julian Groos Verlag, pp. 173198.

NiCKEL, G. 1966. Die Expanded Form im Altenglischen: Vorkommen, Funktion und Herkunft der Umschrebung "Beon/Wesan" + Partizip Präsens. Neumünster: Karl Wachholtz Verlag.

NICKEL, G. 1967. “An Example of a Syntactic Blend in OE”, Indogermanische Forschungen, 72, pp. 261-274.

NúÑEZ-PERTEJO, P. 2004. The Progressive in the History of English with Special Reference to the Early Modern English Period: A Corpus-Based Study. München: LINCOM.

PETRÉ, P. 2015. "Grammaticalization by Changing Co-text Frequencies, or Why [BE Ving] Became the "Progressive", English Language and Linguistics, 20(1), pp. 31-54. https://doi.org/ 10.1017/S1360674315000210. 
PETRÉ, P. 2017. “The Extravagant Progressive: An Experimental Corpus Study on the History of Emphatic [BE Ving]", English Language and Linguistics, 21(2), pp. 227-250. https://doi.org/10.1017/S1360674317000107.

QUIRK, R., Greenbaum, S., Leech, G. y Starvik, J. 1985. A Comprehensive Grammar of the English Language. London: Longman.

Rissanen, M. 1999. "Syntax", en Lass, R. (ed.), Vol. 3 of The Cambridge History of the English Language. Cambridge: Cambridge University Press, pp. 187-326.

SCHEFFER, J. 1975. The Progressive in English. Amsterdam: North Holland-Publishing.

SMITH, K. A. 2007. "The Development of the English Progressive", Journal of Germanic Linguistics, 19(3), pp. 205-241. https://doi.org/10.1017/S1470542707000098.

SMitTerberg, E. 2005. The Progressive in 19th-Century English: A Process of Integration. Amsterdam/New York: Rodolpi.

STRANG, B. 1970. A History of English. New York: Methuen.

Talmy, L. 2000. Toward a Cognitive Semantics. Vol. 2: Typology and Process in Concept Structuring. Cambridge, MA: The MIT Press.

Taylor, A., Warner, A., Pintzuk, S. y Beths, F. 2003. The York-Toronto-Helsinki Parsed Corpus of Old English Prose (YCOE) (1st ed.). Heslington: University of York/Oxford Text Archive. Disponible en: http://www-users.york.ac.uk/ lang22/YcoeHome1.htm.

TRAUGOTT, E. C. 1972. A History of English Syntax: A Transformational Approach to the History of English Sentence Structure. New York: Rinehart and Winston.

TraugotT, E. C. 1990. "From Less to More Situated in Language: The Unidirectionality of Semantic Change”, en Sylvia, A., Law, V., Vincent, N. y Wright, S. (eds.), Papers from the 5th International Conference on English Historical Linguistics. Amsterdam: John Benjamins, pp. 496-517.

Traugott, E. C. 1992. "Syntax", en Hogg, R. (ed.), The Cambridge History of the English Language. Vol. 1. Cambridge: Cambridge University Press, pp. 168-289.

TraugotT, E. C. 2003. “Constructions in Grammaticalization”, en Joseph, B. y Janda, R. (eds.), The Handbook of Historical Linguistics. Oxford: Blackwell, pp. 624-647.

VAN ARKEL-Simón, C. 2018. "Modelos estadísticos para el análisis funcional de los procesos de gramaticalización en las construcciones progresivas del inglés”, Epos: Revista de Filología, 34, pp. 333-355. https://doi.org/10.5944/epos.34.2018.21840.

VAN ARKEL-Simón, C. 2019. "Análisis cualitativo-funcional comparativo para el estudio de los procesos de gramaticalización en las construcciones progresivas del inglés”, Revista de Filología de la Universidad de La Laguna, 39, pp. 71-94. https://doi.org/10.25145/ j.refiull.2019.31.02.

VAN ARKEL-SIMÓN, C. 2020. "Estudio cualitativo de los procesos de gramaticalización, cambio construccional y construccionalización en las construcciones progresivas del inglés", Cuadernos de Investigación Filológica, 48, pp. 163-188. https://doi.org/10.18172/cif.4359.

VISSER, F. T. 1963-1973. An Historical Syntax of the English Language. Vols. 1, 2 y 3. Leiden: E. J. Brill. 


\section{NOTA SOBRE EL AUTOR}

Carlos van Arkel-Simón es Profesor Contratado de Lengua y Lingüística Inglesas en la Universidad de La Laguna (ULL) y Profesor-Tutor de Lengua y Lingüística Inglesas en la Universidad Nacional de Educación a Distancia (UNED). Experto en Lingüística Inglesa y Educación a Distancia, es Doctor en Lengua y Lingüística Inglesas por la UNED. Ha trabajado extensamente en Lingüística Diacrónica y en Lingüística Cognitiva y ha publicado varios artículos científicos en esas áreas de conocimiento. Ha sido también Investigador Colaborador en la Universidad de Córdoba (UCO) en varios Proyectos de Investigación en el ámbito de la Lingüística Cognitiva. ORCID: 0000-0003-4419089X 